\title{
Culture and Sensitivity Pattern of Sepsis in Ventilated Newborns
}

\author{
Sur S ${ }^{1}$, Pallavi ${ }^{2}$, Mondal S ${ }^{3}$, Gupta $\mathrm{AK}^{4}$
}

${ }^{1}$ Dr. Somen Sur, MBBS. MD (Post Graduate Trainee). ${ }^{2}$ Dr. Pallavi, MBBS. MD (Post Graduate Trainee). ${ }^{3} \mathrm{Dr}$. Sanjib Mondal, MBBS. MD (Post Graduate Trainee). ${ }^{3}$ Dr. Atul Kumar Gupta, MBBS. DCh, MD, Professor and Head of the Department. All from the Department of Paediatrics, Vivekananda Institute of Medical Sciences, Kolkata, West Bengal

\section{Address for correspondence:}

Dr. Somen Sur

67/E/2,G.T.ROAD (west),

P.O. Mullickpara, P.S. Serampore, Dist. Hooghly, West Bengal, India, Pin : 712203

Tel No; +919433428988

E-mail: somensur29@gmail.com

Acknowledgements: None

Funding: Nil

Conflict of Interest: None

Permission from IRB: Yes

Ethical dilemmas faced during study: None

\section{How to cite}

Sur S, Pallavi, Mondal S, Gupta AK. Culture and Sensitivity Pattern of Sepsis in Ventilated Newborns. J Nepal Paediatr Soc 2016;36(1):3437.

doi: http://dx.doi.org/10.3126/jnps.v36i1.12936

This work is licensed under a Creative Commons Attribution 3.0 License.

\section{(c) (i)}

\section{Introduction}

\begin{abstract}
Introduction: Neonatal sepsis is a major cause of mortality and morbidity in modern neonatal intensive care units (NICU). Increasing trend of antibiotic resistance makes this problem more difficult to tackle, at present. Haemodynamically compromised neonates, particularly those requiring ventilator support, often suffer from multidrug resistant sepsis. The objectives of this study were to determine micro-organisms causing sepsis in those neonates requiring ventilator support and the sensitivity patterns of those micro-organisms. Material and Method: During 18 months of study period, 48 neonates, who had fulfilled the chosen inclusion criteria were included in this study. They were investigated with blood culture, urine culture, CSF culture, and endotracheal tube tip culture (taken within 48 hours of starting ventilation), as per relevance. Those who were found positive to any micro-organism, were also explored. They were studied further for antimicrobial sensitivity pattern. Data obtained from this study were analysed using SPSS-20.0. Results: 15(31.3\%) neonates were found to be culture positive. Klebsiella sp. was most common (33.3\%) organism isolated, followed by Pseudomonas aeruginosa (20\%). Most common antibiotic found to be sensitive was Meropenem (86.67\%), followed by Colistin and Tigecycline (80\% each). Conclusion: Nowadays, all NICUs throughout the world face a common problem of multidrug resistant sepsis. There is always a doubt regarding choice of second line antibiotics, when one considers for upgradation. As per this study, authors considered Meropenem as second line antibiotic of choice, as other sensitive antibiotics like Colistin, Tigecycline wield more adverse effects on neonates.
\end{abstract}

Key words: meropenem, neonate, resistance, sepsis, ventilation

$\mathrm{N}^{\prime}$ CUs today face one common problem of tackling sepsis, and neonatologists remain constantly baffled by the patterns of microbial floras and their sensitivity patterns ${ }^{1,2,3}$. The morbidity and mortality increase several folds in neonates requiring ventilator 
support for prolonged period ${ }^{4,5,6}$. Very often, one needs to upgrade antimicrobial regimen in these neonates due to poor response to initial antimicrobials ${ }^{4}$. Antibiotic resistance is a major problem in hospitals around the world and this is especially critical in ICU setup ${ }^{7,8}$. In almost all suspected sepsis neonates, antimicrobial therapy is initiated empirically before results of blood cultures are available. It has been realised that the spread of drug resistant organisms in NICU is related to widespread use of antibiotics ${ }^{2,3,9}$. Keeping in mind the high mortality and morbidity associated with neonatal sepsis ${ }^{10,11}$, proper policy regarding empirical antimicrobial strategy is of very much importance and should be individualized according to predominant organisms and their sensitivity patterns of individual NICU of the corresponding institute ${ }^{12,13,14}$.

For the afore-said reason, authors decided to conduct a study with an aim to determine microorganisms commonly involved in causing neonatal sepsis requiring ventilator support, and also to determine sensitivity patterns of those micro-organisms.

\section{Material and Methods}

This study was conducted in the neonatal intensive care unit of Department of Paediatrics, Vivekananda Institute of Medical Sciences on 48 neonates having sepsis requiring ventilator support, over 18 months of the study period. The criteria for neonates to be included in this study had been SEPSIS SCREEN ${ }^{9}$ positive, i.e.

a. Total leukocyte count $<5000 / \mathrm{cmm}$

b. Absolute neutrophil count: Low as per Monroe ${ }^{15}$ chart for term, and as per Mouzinho ${ }^{16}$ chart for very low birth weight neonates

c. Immature neutrophils (band form, myelocytes, metamyelocytes) : total neutrophil (mature + immature) $>0.2$

d. micro ESR $>15 \mathrm{~mm}$ in $1 \mathrm{st} \mathrm{hr}$

e. CRP positive

If two or more parameters were abnormal, it was considered as a positive screen. Thus, only sepsis screen positive neonates $[n=48]$ were included in this study.

Neonates fulfilling the afore-said criteria were investigated with blood culture, urine culture, CSF culture, endotracheal tube tip culture (taken within 48 hours of starting mechanical ventilation), and tracheobronchial aspirate culture (as per relevance with presentation and course of illness). Those found positive for any microorganism growth, were studied further for relevant antimicrobial sensitivity pattern(s). In this institute, Mc-conkey agar, nutrient agar, blood agar, nutrient broth, glucose broth, Sabouraud dextrose agar medium (for fungal culture) were used mainly as culture media and Turbid metric method was used for antibiotic sensitivity testing. Data obtained from this study were entered in Microsoft Excel and subsequently analysed in SPSS version 20.0. Descriptive data were analysed in terms of evaluating simple frequency, percentage, mean, standard deviation (SD), median, I.Q.R., etc. and presented in the following tables.

\section{Results}

Table 1: Distribution of study population according to time of onset of sepsis $(n=48)$.

\begin{tabular}{ccc}
\hline Time of onset of sepsis & Frequency & Percent \\
\hline Early onset & 29 & 60.4 \\
\hline Late onset & 19 & 39.6 \\
\hline Total & $\mathbf{4 8}$ & $\mathbf{1 0 0 . 0}$ \\
\hline
\end{tabular}

In majority of cases (60.4\%), was suffering from early onset neonatal sepsis (Table 1).

Table 2: Distribution of study population according to culture report $(n=48)$

\begin{tabular}{ccc}
\hline Culture report & Frequency & Percent \\
\hline Positive & 15 & 31.3 \\
Negative & 33 & 68.8 \\
\hline Total & $\mathbf{4 8}$ & $\mathbf{1 0 0 . 0}$ \\
\hline
\end{tabular}

In $31.3 \%$ of cases, culture report was found to be positive (Table 2).

Table 3: Distribution of study population according to organisms found in culture report $(n=15)$

\begin{tabular}{lcc}
\hline Organism & $\begin{array}{c}\text { Frequency } \\
(\mathbf{n = 1 5 )}\end{array}$ & $\begin{array}{c}\text { Percent } \\
(\mathbf{\%})\end{array}$ \\
\hline Klebsiella sp & 5 & 33.3 \\
\hline $\begin{array}{l}\text { Pseudomonas } \\
\text { aeruginosa }\end{array}$ & 3 & 20.0 \\
\hline E.coli & 2 & 13.3 \\
\hline Acinatobacter baumannii & 2 & 13.3 \\
\hline CONS & 2 & 13.3 \\
\hline Group B Streptococci & 1 & 6.67 \\
\hline Total & $\mathbf{1 5}$ & $\mathbf{1 0 0 . 0}$ \\
\hline
\end{tabular}

Most common organism found in culture (Table 3) was Klebsiella sp (33.3\%) followed by Pseudomonas aeruginosa (20\%). 
Table 4: Distribution of study population according to sensitivity pattern of organisms to antibiotics $(n=15)$

\begin{tabular}{ccc}
\hline Antibiotics* $^{*}$ & Frequency & Percent \\
\hline Meropenem & 13 & 86.67 \\
\hline Colistin & 12 & 80.0 \\
\hline Tigecycline & 12 & 80.0 \\
\hline Vancomycin & 3 & 20.0 \\
\hline Linezolid & 3 & 20.0 \\
\hline Cefepime & 1 & 6.67 \\
\hline Ceftazidime & 1 & 6.67 \\
\hline${ }^{*}$ not mutually exclusive] & &
\end{tabular}

Most common antibiotics found to be sensitive against the organisms found in culture (Table 4) were meropenem $(86.67 \%)$, colistin and tigecyclin (80\%).

\section{Discussion}

Sepsis is one of the most common causes of mortality and morbidity in neonatal population. Today, the NICUs throughout the globe are facing multiple obstacles in handling this problem like changing pattern/s of causative organisms, and more importantly, widespread resistance of common antimicrobials by these organisms. In developing countries, gram negative organisms are more common and mainly consist of Klebsiella $s p$, E.coli and Pseudomonas $s p^{1,11,17}$. Among gram positive organisms, Staphylococcus aureus, coagulase negative Staphylococcus (CONS) and Streptococcus pneumoniae are most common ${ }^{1,11}$. On studying antibiotic sensitivity pattern, it was found that most of Klebsiella sp and E.coli were resistant to ampicillin and gentamycin ${ }^{7,8}$. High proportion of gram positive bacteria were found to be resistant to cloxacillin. A study from CMC, Vellore showed that E.coli was usually sensitive to cefotaxime, gentamycin and amikacin. But, Klebsiella $s p$ and Enterobacter sp,which were responsible for most late onset of neonatal sepsis [LONS], were resistant to aminoglycosides and cephalosporins, and sensitive only to ciprofloxacin and imipenem ${ }^{18}$. Indian units including that in Bangalore reported a predominance of Gram negative organisms like Klebsiella sp, Pseudomonas $s p$, etc. and Gram positive bacteria like Staphylococcus aureus ${ }^{19}$. The centres abroad reported sepsis with Group B Streptococcus and coagulase negative Staphylococcus ${ }^{20}$. In this study also, most prevalent offending organism was found to be Klebsiella $s p$, followed by Pseudomonas $s p$.

As per study conducted by Naher and Khamel ${ }^{21}$, $58 \%$ neonates were detected with early onset neonatal sepsis [EONS] and 42\% neonates were with LONS.
The results indicated that the incidence of EONS was more common than that of LONS. In the present study, $29(60.4 \%)$ babies were suffering from early onset of neonatal sepsis, comparable with that study. It is also comparable with the reports from other developing countries like Iran (77.5\% vs $22.5 \%)^{22}$ and Bangladesh $(70.7 \% \text { vs } 29.3 \%)^{23}$.

Results of culture positive sepsis by various authors like Dias et al. ${ }^{24}$, Shaw et al. ${ }^{25}$, Bhattacharjee et al. ${ }^{26}$ were $32 \%, 54.64 \%$ and $48 \%$ respectively. They are comparable with the present results $(31.3 \%)$. This result is also comparable with the study conducted by Shah et al. ${ }^{27}(31.57 \%)$.

National Neonatology Forum of India expresses their concern regarding the fact that there are exceedingly high rate of resistance of Gram negative bacilli to almost all antibiotics. Resistance to aminoglycosides is about $50 \%$ for amikacin, higher for netilmicin and over $75 \%$ for gentamicin. Resistance to third generation cephalosporins is $80 \%$ plus. Bacteria are less resistant $(30-46 \%)$ to piperacillin-tazobactam. Imipenem resistance is already appearing (about $20 \%)^{28,29}$.

Mahmood et al. ${ }^{30}$ had also showed in their study that more than $90 \%$ gram negative rods had been resistant to ampicillin and co-trimoxazole. Resistance to gentamicin was as high as $90.4 \%$ for Klebsiella pneumoniae, and $60.87 \%$ for Acinetobacter baumannii. Resistance to the third generation cephalosporins and the quinolone tested (ciprofloxacin) varied between $25-75 \%$. Majority of the isolates were susceptible to meropenem and amikacin.

In the present study also, the predominant organisms in babies suffering from severe neonatal sepsis were gram negative rods [Klebsiella sp (33.3\%), Pseudomonas sp (20\%)] and they were mostly resistant to first line antibiotics and sensitive to higher antimicrobials like meropenem (86.67\%), colistin, tigecycline ( $80 \%$ each), comparable to afore-said studies.

The main limitation of this study is small study population, limited period of study and study population covering only hospital based babies. Further large scale study should be conducted to get a more detailed knowledge regarding this issue.

\section{Conclusion}

As per the present study, Klebsiella sp. was found to be the most common organism contributing in severe 
neonatal sepsis in this hospital. But, since there is a confusion when upgrading the antibiotics, authors found that they could consider Meropenem as second line antibiotic of choice because other antimicrobials like colistin and tigecycline wield more adverse effects on neonates.

\section{References}

1. Deorari AK, Paul VK, Agarwal $R$ et al. Changing pattern of bacteriologic profile in neonatal sepsis among intramural babies. J Neonatol 2006;20:8-15.

2. Dutta S, Kadam S, Saini SS et al. Management of Neonatal Sepsis. NNF Clinical Practice Guidelines, 2010:155-71.

3. Viswanathan $R$, Singh AK, Ghosh $C$ et al. Profile of Neonatal Septicemia at a District -level Sick Neonatal Care Unit. J Health Popul Nutr 2012;30(1):41-48.

4. Bozaykut A, Ipek IO, Kilic BD. Predicting Neonatal Sepsis in Ventilated Neonates. Indian J Pediatr 2008; 75: $39-42$.

5. Fogila E, Meier MD, Elward A. Ventilator Associated Pneumonia in Neonatal and Pediatric Intensive Care Unit Patients. Clin Microbiol Rev 2007;20(3):409-25.

6. Garland JS. Strategies to Prevent Ventilator Associated Pneumonia in Neonates. Clin Perinatal 2010;37(3):629-643.

7. Johnson PJ. Antibiotic Resistance in the NICU. Neonatal Netw 2012 Mar-Apr; 31(2) : 109-14.

8. Najeeb S, Gillani S, Rizvi SK et al. Causative Bacteria and Antibiotic resistance in neonatal sepsis. J Ayub Med Coll Abbottabad 2012;24(3-4):131-34.

9. Sankar MJ, Agarwal R, Deorari AK et al. Sepsis in the Newborn, Symposium on AlIMS Protocols Neonatol-3, 2008:261-266.

10. Carcillo JA. Pediatric septic shock and multiple organ failure. Crit Care Clin 2003;19:413-440.

11. Deorari AK, Paul VK, Agarwal R et al. Report 20022003. National neonatal perinatal database network, New Delhi, National Neonatology Forum of India, 2004: 6-52.

12. Patel SJ, Saiman L. Antibiotic Resistance in NICU Pathogens : Mechanisms, clinical impact, and prevention including Antibiotic Stewardship. Clin Perinatol 2012;39(1):61-68.

13. Suryawanshi P. Antibiotic usage and Rational guideline practices in neonatal sepsis. VIMS Health Sci J 2014;1:33-40.

14. Aguilar CY, Maramba-Lazarte CC. A cross sectional analysis of neonatal bacteraemia in the neonatal intensive care unit of the Philippine general hospital from July to December 2006. Pediatr Infect Dis Soc Philippines J 2011;12(1):17-27.

15. Manroe BL, Weinberg AG et al. The neonatal blood count in health and disease. Reference values for neutrophilic cells. J Pediatr 1979;95(1):89-98.
16. Mouzinho A, Sanchez PJ et al. Revised reference ranges for circulating neutrophils in very low birth weight neonates. J Pediatr 1994;94(1):76-82.

17. Bates M, Kabwe M, Zumla A. Neonatal Sepsis and Antibiotic Resistance in developing countries. Pediatr Infectious Dis J 2014;33(10):1097.

18. State of India Newborn, National Neonatology Forum, India; Save the Children, USA in November, 2004.

19. Chacko B, Sohi I. Early onset neonatal sepsis. Indian J Pediatr 2005;72(1):23-26.

20. Rasaily R. Epidemiology of neonatal infections: community experience. J Neonatol 2009;23(1):12-21.

21. Naher HS, Khamael AB. Neonatal Sepsis : The Bacterial causes and the Risk factors. Int Res $J$ Medical Sci 2013;1(6):19-22.

22. Movahedian A.H., Moniri R. and Mosayebi Z. Bacterial culture of neonatal sepsis. Iranian J Publ Health 2006;35: 84-89.

23. Rasul C.H., Hassan M.A. and Habibullah M. Neonatal sepsis and use of antibiotic in tertiary care hospital. Pak J Med Sci 2007;23:78-81.

24. Dias $E$, Vighneshwaran $P$. The bacterial profile of neonatal septicaemia in a rural hospital in south India. J Clin Diagn Res 2010; 4 : 3327-30.

25. Shaw CK, Shaw P, Thapalial A. Neonatal sepsis bacterial isolates and antibiotic susceptibility patterns at a NICU in a tertiary care hospital in western Nepal: A retrospective analysis. Kathmandu Univ Med $J$ 2007;5:153-60.

26. Bhattacharjee A, Sen MR, Prakash P, Gaur A, Anupurba $S$. Increased prevalence of extended spectrum $\beta$-Lactamase producers in neonatal septicaemic cases at tertiary referral hospital. Indian J Med Microbiol 2008;26:356-60.

27. Shah AJ, Mulla SA, Revdiwala SB. Neonatal Sepsis: high antibiotic resistance of the bacterial pathogens in a neonatal intensive care unit of a tertiary care hospital. J Clin Neonatal 2012;1(2):72-75.

28. Isaacs D. Neonatal sepsis: the antibiotic crisis. Indian Pediatr 2005;42:9-13.

29. Tripathi N, Cotton CM, Smith PB. Antibiotic use and misuse in the neonatal intensive care unit. Perinatology 2012;39(1):61-68.

30. Mahmood A et al. Neonatal Sepsis: High Antibiotic Resistance of the Bacterial Pathogens in a Neonatal Intensive Care Unit in Karachi. JPMA 2002;52:348. 